\title{
Image classification for the painting style with SVM
}

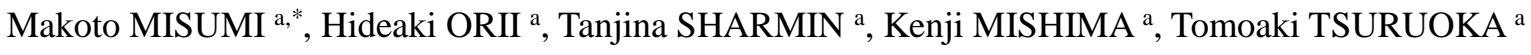 \\ a Faculty of Engineering, Fukuoka University, 8-19-1 Nanakuma, Jonan-ku, Fukuoka 814-0180, JAPAN \\ *Corresponding Author: mmisumi@fukuoka-u.ac.jp
}

\begin{abstract}
Classification of the painting style of painting by a computer is required. The painting style of painting is characterized by such as colors, brushstroke and texture of the painting. When we classify the huge data of the painting, we should recognize the painting style of painting by using an image recognition method. In this study, we discuss a new approach to achieve the objective classification and identification of the painting style of paintings using the image recognition approach. "Bag of Visual-words" was used in this work. That has been widely used in the field of image recognition. At the learning of the feature, Support Vector Machine (SVM) was used as the machine learning method. In the experiment, accuracy of the classification attains $72 \%$.
\end{abstract}

Keywords: Bag-of-visual-words, Painting style, Cubist painting

\section{Introduction}

Recently, it has been required to record the many traditionally important painting as digital data in order to keep quality of culture. Computer aided classification of painting style of painting has been expected to be developed and many researchers have reported some methods. In this study, we have focused on classifying a Cubist painting and other painting, such as Realism painting, because many artificial patterns, such as triangle, are included in the Cubist painting. An example of Cubist painting is shown in Fig.1. A new approach to classification and identification of the

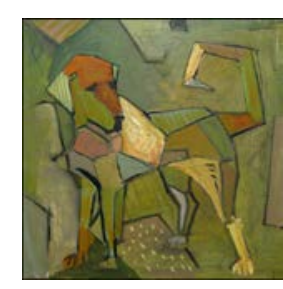

Fig. 1 Example of Cubist painting ${ }^{13)}$

painting style of paintings has been examined by means of the image recognition. "Bag of Visual-words", which has been widely used in the field of image recognition, was applied to the pattern recognition of paint style of painting in this work. At the learning of the feature for the painting, Support Vector Machine (SVM) ${ }^{(4-7)}$ was used as the machine learning method.

\section{Method}

\subsection{Bag of Visual-words and SIFT}

We utilized Scale-invariant feature transform $(\mathrm{SIFT})^{(2,8 \text {, }}$ $10,11)$ as a computer algorithm in order to detect and describe local features in images of painting vision data. Bag of Visual-words (BoVW) was applied to basic image system. (1-3) Fig.2 shows an overview of the recognition process with BoVW. Interesting points on the object of painting image data can be extracted to provide a histogram that represents the frequent degree of pattern in the object of painting. This histogram, extracted from a training image, can be utilized to identify the object of painting when attempting to locate the object in a test image. 


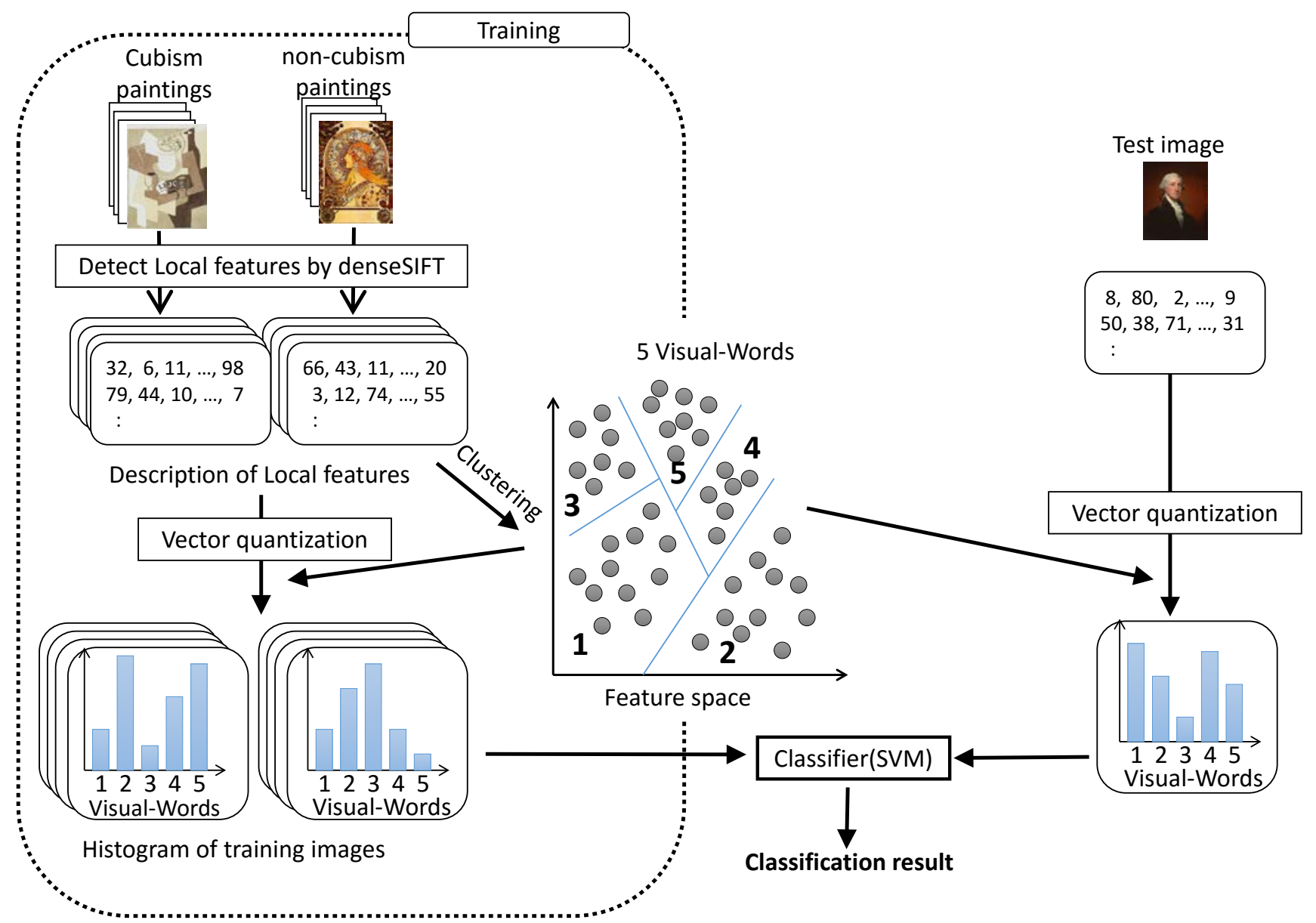

Fig. 2 Overview of bag-of-visual-words.

\subsection{Local feature}

SIFT feature is a local feature quantity described by the intensity gradient of the image. SIFT is a local feature descriptor invariant to rotation and intensity change. As shown in Figure 3, the SIFT to select the point on the image as a key point, and to select a rectangular region of a particular size centered on the key points. Then, selected rectangular region divided into 16 small rectangular region.

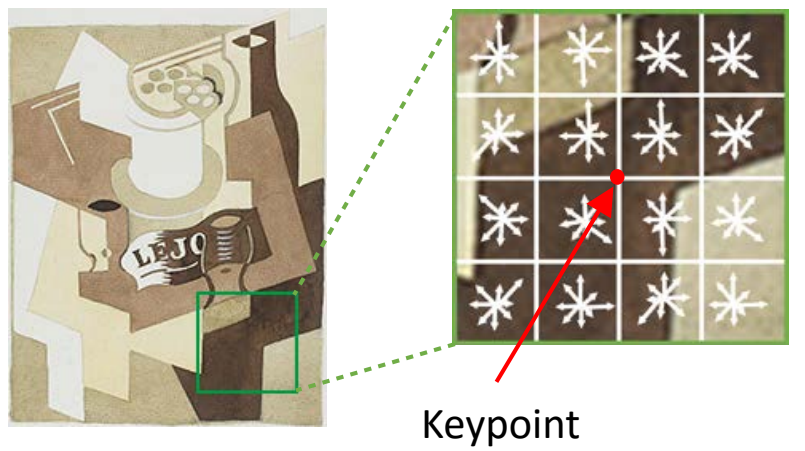

Fig. 3 SIFT descriptor (Example of Cubist painting ${ }^{12)}$ )
The weighted histogram of the 8-directions gradient strength of each small rectangular region is calculated. Concatenation of the weighted histogram of the 8-directions gradient strength is the local features. The orientation of the region around the key point is calculated from the gradient intensity distribution of the entire region. Local feature becomes invariant to a rotation by performing normalization of the orientation by the orientation in the feature description.

Gradient strength $\mathrm{m}(\mathrm{u}, \mathrm{v})$ of the image I $(\mathrm{u}, \mathrm{v})$ and the gradient direction $\theta(\mathrm{u}, \mathrm{v})$ is calculated by the following equation.

$$
\begin{gathered}
\mathrm{m}(\mathrm{u}, \mathrm{v})=\sqrt{f_{u}(u, v)^{2}+f_{v}(u, v)^{2}} \\
\theta(\mathrm{u}, \mathrm{v})=\tan ^{-1} \frac{f_{u}(u, v)}{f_{v}(u, v)} \\
\left\{\begin{array}{l}
f_{u}(u, v)=I(u+1, v)-I(u-1, v) \\
f_{v}(u, v)=I(u, v+1)-I(u, v-1)
\end{array}\right.
\end{gathered}
$$

Gradient strength $\mathrm{m}(\mathrm{x}, \mathrm{y})$ of the key points surrounding region and the gradient direction $\theta(\mathrm{x}, \mathrm{y})$ was used to 
calculate a weighted orientation histogram of the gradient strength $h$.

$$
\begin{gathered}
\mathrm{h}_{\theta^{\prime}}=\sum_{x} \sum_{y} w(x, y) \cdot \delta\left[\theta^{\prime}, \theta(x, y)\right] \\
\mathrm{w}(\mathrm{x}, \mathrm{y})=\mathrm{G}(\mathrm{x}, \mathrm{y}, \sigma) \cdot \mathrm{m}(\mathrm{x}, \mathrm{y})
\end{gathered}
$$

Where $\mathbf{h}_{\theta}$ is a quantized histogram from all directions to 36 directions. $\mathrm{w}(\mathrm{x}, \mathrm{y})$ is the weight of the pixel $(\mathrm{x}, \mathrm{y})$ in the local region. $w(x, y)$ is calculated from Gaussian window $G(x, y$, $\sigma)$ and the gradient strength $m(x, y)$. The scale of $G(x, y, \sigma)$ is equivalent to the size of the key points around region. $\delta$ [ , ] is the delta function of Kronecker. When the gradient direction $\theta(x, y)$ is included in the direction $\theta^{\prime}$ that has been quantized, the function $\delta$ [ , ] returns 1 . Assign a peak, which is at least $80 \%$ from the maximum value of 36 direction histograms, in the key point of orientation. By weighting using a Gaussian window, gradient strength in which the pixel close to the key point has to act strongly against the determination of the orientation.

SIFT feature is described based on the detected orientation.

\subsection{Creating a Visual-Words dictionary}

A Visual-Words dictionary was created by accumulation of the local features obtained from the local image patterns that frequent in the image of painting by using a large number of the general image of painting. In our work, the local image patterns were extracted with the dense $\operatorname{SIFT}^{(2,8,9)}$ from a number of painting images randomly selected. Visual cluster centers were accumulated as an element of the Visual-Words dictionary, which obtained by performing clustering using the k-means algorithm based on the total feature value.

\subsection{Histograms of painting style}

Histograms of the local feature quantities for painting style of a certain painting were obtained from the local feature quantities calculated from the local image patterns with the dense SIFT using the created Visual-Words dictionary.

\subsection{Classification with Support Vector Machine}

Classification of the certain painting was done with Support Vector Machine (SVM) using the histogram of the local feature quantities for painting style of the painting. The SVM is a supervised learning model used in machine learning with associated learning algorithms that analyze data and recognize pattern. It provides the classification of images using histograms of the local feature quantities.

\subsection{Cross-validation of classification}

Cross-validation of classification for the painting style was carried out. 18 Cubist paintings, 11 Art Nouveau paintings, and 13 paintings of expressionism school were used as image data

\section{Experimental Results and discussions}

The purpose of this study was classification of the painting style of painting. We compared the histogram as the image feature and then we evaluated the painting style classification accuracy of the proposed method. In section 3.1, we will explain about the visual comparison of the histogram as the image feature. In section 3.2, we show the results of the proposed method, and then discuss the results.

\subsection{Visual comparison of the histograms as the image feature}

In this chapter, we will consider whether the histogram of the image, which is a feature of BoVW is different from how each painting style.We obtained many histograms for

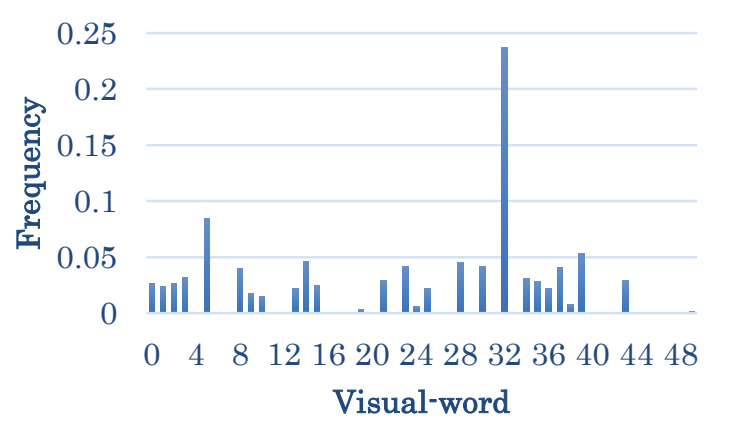

(a) Cubist painting

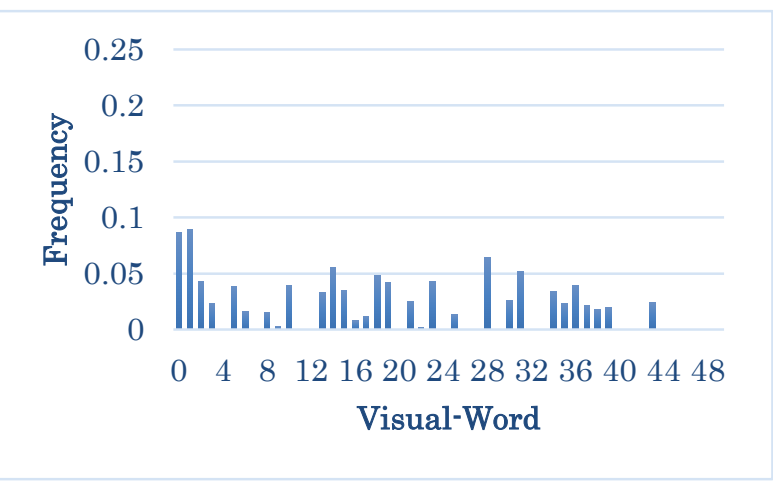

(b) Art nouveau painting.

Fig. 4 Example of visual-words histogram. 


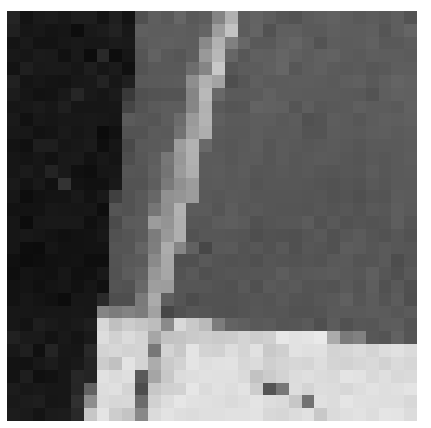

Fig. 5. The 32nd visual-word.

painting style, such as Cubist paintings, Art Nouveau paintings, and Paintings of expressionism. Examples of histograms for painting style of painting obtained from the local feature quantities calculated from the local image patterns with the dense SIFT for Cubist paintings and Art Nouveau paintings are shown in Fig. 4. Shown in Fig. 4(a) and Fig. 4(b), the 32nd Visual-Word has been frequent in the Cubist image. As shown in Fig. 5, the 32nd Visual-word is a linear pattern that represents the characteristics of the cubist painting. From the above, the histogram clearly shows the characteristics of each style of painting.

\subsection{SVM Classification result}

As shown in Table 1, we have collected 42 painting images a dataset for 2 -fold cross validation. The width of all images resized to 200 pixels. We have set the key point in the 1 pixel spacing, and we use three scales (32, 45 and 64 pixels). And we have created 10, 100 and 500 Visual-Words dictionary using the k-means. We have 2-fold cross

Table 1. Image dataset.

\begin{tabular}{|c|c|c|c|}
\hline Positive & \multicolumn{2}{|c|}{ Negative } & \multirow{2}{*}{ Total } \\
\cline { 1 - 3 } Cubist & $\begin{array}{c}\text { Art Nouveau } \\
\text { painting }\end{array}$ & $\begin{array}{c}\text { Realism } \\
\text { painting }\end{array}$ & \\
\hline 18 & 11 & 13 & 42 \\
\hline
\end{tabular}

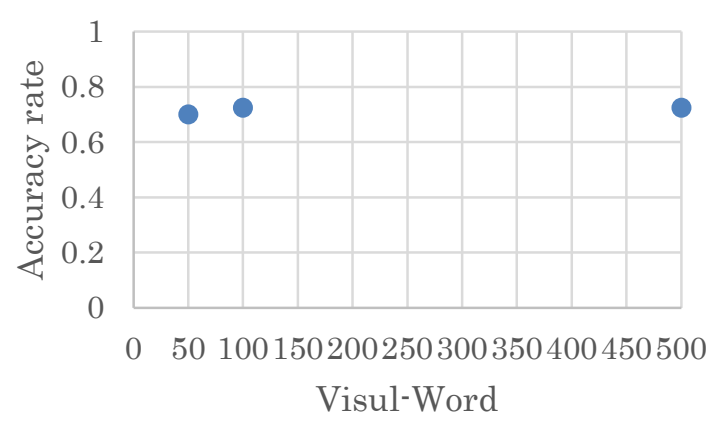

Fig. 6. Results of cross-validation. validation for each Visual-Word dictionary in the SVM using the RBF kernel. Fig. 6 shows accuracy of classification by size of Visual-Word. As shown in Fig. 6, when the size of the visual-words is 50 , classification accuracy is $70 \%$. While when the size of the word is more than 100, classification accuracy is $75 \%$. This result indicate that the proposed method achieve sufficient classification performance.

\section{Conclusions}

In this paper we proposed a new approach to achieve the objective classification and identification of the painting style of paintings using the image recognition approach. We used "Bag-of-visual-words" as the feature of painting style, and used SVM for classification. We have verified that the accuracy rate of the proposed method is more than $70 \%$.

\section{Acknowledgement}

This research was partially supported by a Grant-in-Aid for Scientific Research (Grant Nos. 26420770 and 23560913). The authors gratefully acknowledge the Japan Food Chemical Research Foundation (2015).

\section{References}

(1) Csurka, G., Dance, C.R., Fan, L., Willamowski, J., Bray, C.: "Visual categorization with bags of keypoints", European Conference on Computer Vision (ECCV2004) Workshop on Statistical Learning in Computer Vision, pp. 59-74, 2004.

(2) Nowak, E., Jurie, F., Triggs, B.: "Sampling strategies for bag-of-features image classification.”, Computer Vision ECCV-2006, Lecture Notes in Computer Science, vol. 3954. Springer, Berlin/Heidelberg, pp. 490-503, 2006.

(3) Lazebnik, S., Schmid, C., Ponce, J.: "Beyond bags of features: Spatial pyramid matching for recognizing natural scene categories.”, IEEE Computer Society Conference on Computer Vision and Pattern Recognition, pp. 2169-2178, 2006.

(4) Vapnik, V.N: “Statistical Learning Theory.”, Wiley, New York, 1998.

(5) John Shawe-Taylor, N.C.: “An Introduction to Support Vector Machines: And Other Kernel-based Learning Methods.”, Cambridge University Press, Cambridge, New York, 2000.

(6) Schölkopf, B., Smola, A.J.: "Learning with kernels: 
support vector machines, regularization, optimization, and beyond.”, MIT Press, Cambridge, Mass, 2002.

(7) Steinwart, I., Christmann, A.: "Support Vector Machines”, Springer, New York, 2008.

(8) Fei-Fei, L., Perona, P.: "A bayesian hierarchical model for learning natural scene categories.”, IEEE Computer Society Conference on Computer Vision and Pattern Recognition, 2005. CVPR 2005, pp. 524-531, 2005.

(9) Bosch, A., Zisserman, A., Muoz, X.: "Image classification using random forests and ferns.", IEEE 11th International Conference on Computer Vision, 2007. ICCV 2007, pp. 1-8, 2007.

(10) Jurie, F., Triggs, B.: "Creating efficient codebooks for visual recognition.”, Tenth IEEE International Conference on Computer Vision, 2005. ICCV 2005, pp. 604-610, 2005.

(11) Herve, N., Boujemaa, N., Houle, M.E.: "Document description: what works for images should also work for text?”, Multimedia Content Access: Algorithms and Systems III, SPIE, pp. 72550B-72550B-12, 2009.

(12) Juan Gris: "Still Life”, Art Institute of Chicago, 1919.

(13) Emanuel M Ologeanu: "El Vagabundo - cubist painting”. 